\title{
ARTIFICIAL REASONERS FOR HUMAN PROCESS SUPERVISION
}

\author{
Andreu Català, Pere Ponsa \\ Departament d'Enginyeria de Sistemes, Automàtica i Informàtica Industrial \\ UPC \& LEA-SICA \\ Av. Víctor Balaguer, $\mathrm{s} / \mathrm{n}$ \\ 08800 Vilanova i la Geltrú \\ e-mail: \{andreu,pponsa\}@esaii.upc.es \\ Louise Travé-Massuyès \\ LAAS-CNRS \& LEA-SICA \\ 7, Avenue du Colonel-Roche \\ 31077 Toulouse Cedex \\ e-mail:louise@laas.fr
}

\begin{abstract}
A methodology for analysing human reasoning when performing a supervision task has been proposed in WAHRPS project (Worlds for assessing human reasoning in process supervision), conducted by INSERM 455 [1]. The main issue is the modelling of different human reasoning styles implemented as Reference Artificial Reasoners (RAR), and the study of decision sequences in front of several dynamic processes. In this paper, an adaptive tool able to generate different types of dynamical systems with increasing difficulty degrees is presented. The design and implementation of a reference artificial reasoner (RAR) based on sequential control, and the implementation of a RAR based on qualitative reasoning is also reported. Finally, a discussion about the comparison between human and artificial decision sequences, in front of the same dynamical process, is included.
\end{abstract}

\section{Introduction}

The different capacities of human intelligence for process supervision, like making decisions, anticipation, or diagnosis are difficult to study. It requires combined expertise in psychology and artificial intelligence, among other science fields. In this work, the complex process supervision is performed from the point of view of human reasoning: to observe, to validate, and to act. For example, if it is possible to classify different behaviours in front of similar situations, through the study of the evolution of these behaviours, can be diagnosed anomalous situations like fatigues or stress.
The first step is the creation of a set of dynamic systems with diverse degree of difficulty, easy to understand and easy to use: anybody, with or without knowledge of control theory of dynamic systems, should be able to understand and to intuitively control the system in a few seconds. The question is: which is the ideal physic system to study? And, which are the set of rules for operating it?

Within the framework of the WAHRPS project, (Worlds for Assessing Human Reasoning in Process Supervision), with medical application[1], uses micro-worlds based on tanks interconnected with pipes and controlled with binary valves. Our work build in the same type of micro-world. The micro-world is interpreted like a simulation of dynamical 
systems, controlled by human operators, and data extraction capabilities.

The generation of micro-worlds is done with LabVIEW, a versatile software with graphical programming capabilities and near to the industrial reality [2].

To control the binary valves of the microworld, it is possible the use of PC mouse. A second option is an adaptive switch box. A third option is the use of a touch-sensitive screen. The type of the peripheral to use for the control depend on the type of operator (engineering student, industrial operator, other user).

Different paradigm can be at the basis of the design of automatic artificial reasoner.. For example: sequential control, rule based reasoning, neural networks, fuzzy reasoning, qualitative reasoning. Each of the above mentioned paradigm could modelize different reasoning styles, or the same methodology could cover different cognitive styles thought a learning or adaptive process. In that sense, neural networks or fuzzy systems seem to be more capable to deal with intuitive reasoning or implicit reasoning. On the other hand qualitative or rule based reasoning seem to be designed to modelize explicit reasoning. [13]

These are the basic ideas underlying our approach. The next paragraph shows the state of art of our study.

\section{Micro-world framework}

The design of a micro-world to test human behaviour needs to be realistic. In the case of the tanks micro-world, "realistic" means that the water should flow into the tanks in an approximately continuous fashion and that the proportion between tank areas, pipe diameters and fill up velocities are correct. To accomplish this realistic dynamics we use an approximate model of flux equations from classical fluid mechanics [3].

In order to fit into this study, it was decided to implement a micro-world generator with
LabVIEW [2]. Some of the interesting properties of LabVIEW are: assisted modelling facility, flexibility on graphical objects and their parameters, facilities for building real time applications, data extraction and display. Due to these characteristics, we are able to design and modify micro-worlds in an easy way.

\subsection{Modelling with LabVIEW}

There are two important facts. The first one: the mathematical model is an approximation of reality. The second one: the human operator performing supervision tasks uses a mental model without numerical information [9]. Hence, the mental model is also an approximation of reality. The human operators look tendencies, threshold overshoots and, finally make decisions in terms of that actions on valves.

If we want to create a generator of microworlds we should considerate the above facts. From the first one we take basically physical laws. From the second one we will create a realistic simulation. So, we take into account the following approximations:

- We don't work with differential equations. We work with flow (liters per second) and with the fact that the number of liters is constant in the chosen micro-world.

- We don't create a industrial plant. Then, we approximate the valve's behaviour a switch $\mathrm{ON} / \mathrm{OFF}$

- We don't considerate the length of the pipes

\section{Flow and liters' number}

The human operators see height tendencies but it is more appropriate for our purpose, to work with the number of liters. A set of steps is presented:

- Translate height to number of liters

- Look at the valve's state

- At instant k, we calculate

$$
\begin{gathered}
\text { Final liters }(k)=\text { initial liters }(k-1)-\text { output } \\
\text { liters }(k)+\text { input liters }(k)
\end{gathered}
$$


- Addition of output liters with the number of liters of the tank interconnected with the last tank

Each calculation is created with the $G$ source code of LabVIEW and with the design of Virtual Instruments subprograms VI's [7]. LabVIEW allows to set an internal mechanism to update the display according to the system's state changes (for example, water motion). For the micro-world of Figure 1, we can see a source tank with 3 pipes, a intermediate tank with 1 input pipe and 2 output pipe, and a sink tank with 3 input pipes... all these possibilities are tabulated in VI subprograms.

\section{Pipe's flow: a mathematical simplification}

In LabVIEW it is not possible the model pipes geometry [7]. Some authors [6] define the pipe length as the diagonal length between two connected tanks. Flow time between tanks, is this previous defined distance per flow. With LabVIEW this time is smaller than the time to fill or to empty the tank, and smaller that the time that the operator needs to make a control action. So we do not consider pipes geometry in this type of micro-world. On the other hand, LabVIEW shows sufficient industrial realism: different diameters of pipes permit different velocities to fill one tank. Human operator could estimate this speed change and make decisions.

\subsection{Pannel Interface}

We have seen in the previous paragraph the internal face of LabVIEW, also called Diagram, necessary for the internal construction of microworlds. On the other hand, human operators see another interface, called Pannel. In this pannel, the designer builds the micro-world through a library of elements like tanks, pipes, valves, pumps (see Figures 1, 2 and 3). Each element of the library have an internal connection with the subprograms VI's designed by Diagram interface.

Figure 1 shows a micro-world with LabVIEW: it is a hydraulic system with five tanks and pipes of diverse diameters. There is another virtual tank called tank 6 , which is the tank of lost water during the test. Tanks pipes, and binary control valves (on/off) are clearly displayed. This is the interface presented to humans. The capacity of the top tank is the same as the bottom tank. Other types of microworlds can be easily designed. For example, a micro-world with a pump, moving water from bottom tank to top tank, (Figure 2). In this case, capacities of top tank and bottom tank are not equals.

Using the same methodology we could design a micro-world based on the simultaneous control of the evolution of three variables having only the possibility to increase or decrease its values

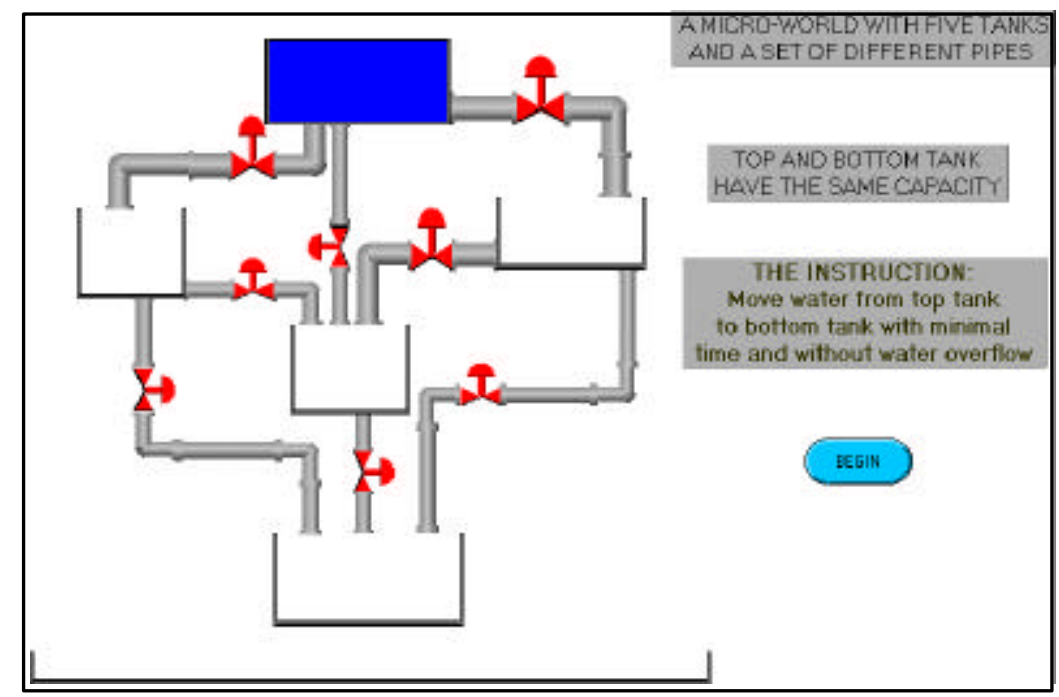

Figure 1: A micro-world example 


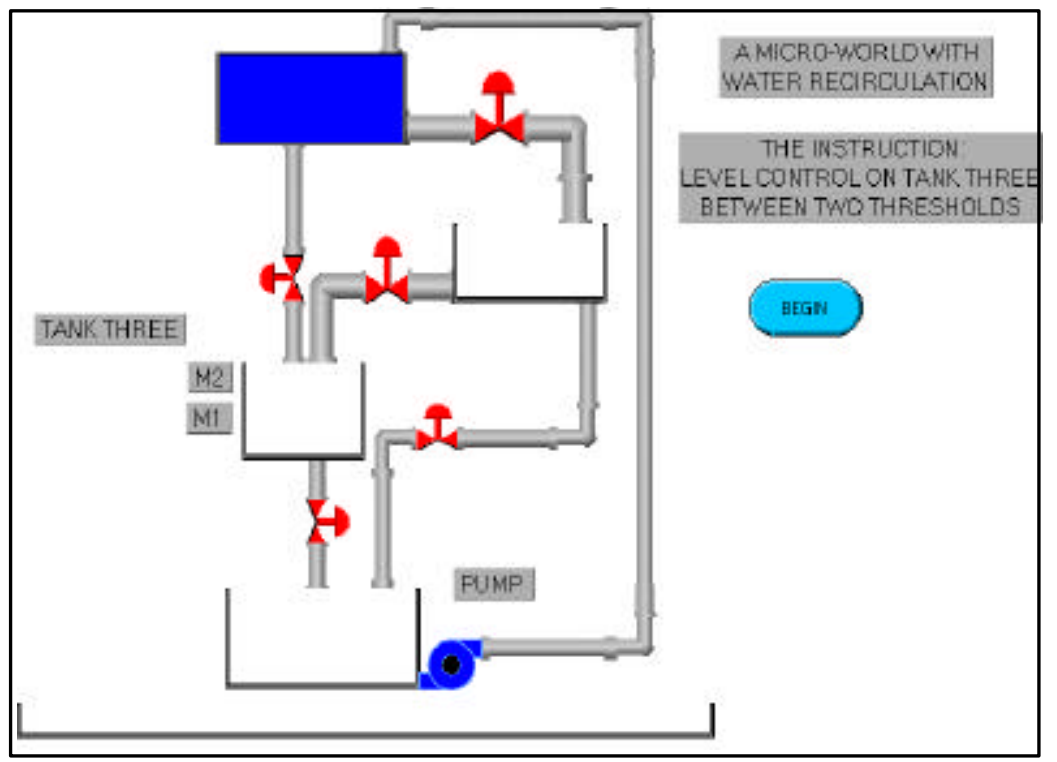

Figure 2: A micro-world with water recirculation

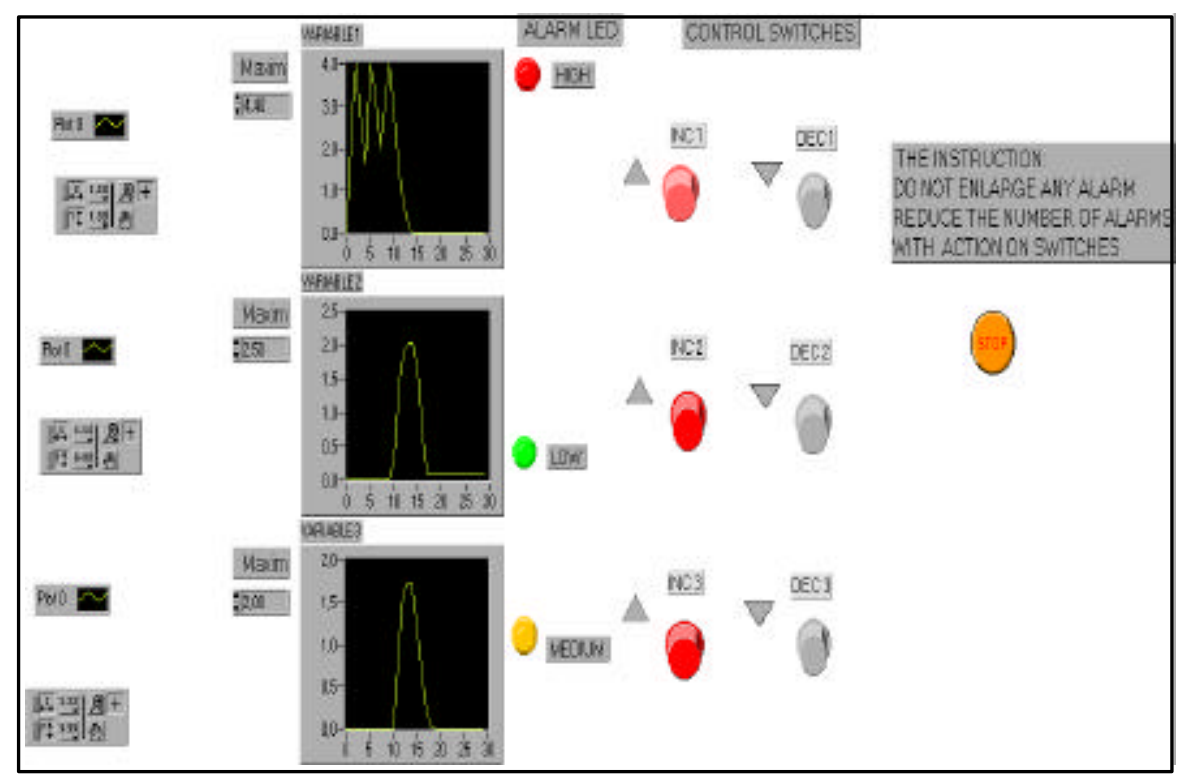

Figure 3: A micro-world with a alarm situation

and with the final objective of not overpassing a previously fixed threshold (Figure 3 ).

Once we have designed, and actually also during the design of a micro-world, it is necessary to have in mind which type of test we would like to do with the final users. We can organise a session test with a set of microworlds with increasing difficulty degree. For example, in the first micro-world all the pipes have the same diameter, and in the next one there are pipes of different diameters. It is necessary to provide at the beginning of the test, a precise explanation about the objectives and the interaction between the micro-world and the user. 


\section{Artificial Reasoners}

Let's imagine that we have an extensive data base of different users, or the same user in different situations (to detect stress, fatigue), or the same user with a prescribed temporisation (medical domain). Our issue is to recognise different types of reasoning strictly coupled with the behaviour presented by the users in the session test. One possible way is to design a set of artificial reasoners (AR) in order to compare each of them with the human behaviour data [1]. The set of AR could be based on different paradigms, but another line of work could be the utilisation of only one paradigm, like for example neural networks, and try to extract from the data base common actions, regularities, pseudo-frequency actions and so on, in order to identify different behaviours. An application of this approximation could be into the behavioural cloning of industrial control operators [11].

At this moment, two representations have been defined corresponding to different possible expertise levels and cognitive strategy:

- Sequential control reasoner (SCR) [7]

- Qualitative reference artificial reasoner (QRAR) [8]

\subsection{Implementation of a sequential control SCR}

Using common sense, we can defined an elementary set of rules for one tank with two level sensors (Figure 4):

- If both sensors are inactive, then open the top valve

- If both sensors are active, then close the top valve and open the bottom valve

- If low height level sensor is active and high level sensor is inactive, then maintain the situation

The SCR with the approximation of sequence control, can be act in mode off line or mode on line. The micro-world software is flexible, an operator can make the test, or the artificial reasoner can make the test, this is the meaning of mode off line. The mode on line is more complex: an operator makes a decision and opens or closes one valve, at the same time, the artificial reasoner shows which type of action is optimal according to its cognitive strategie. This comparison is effective through each step of time along the test.

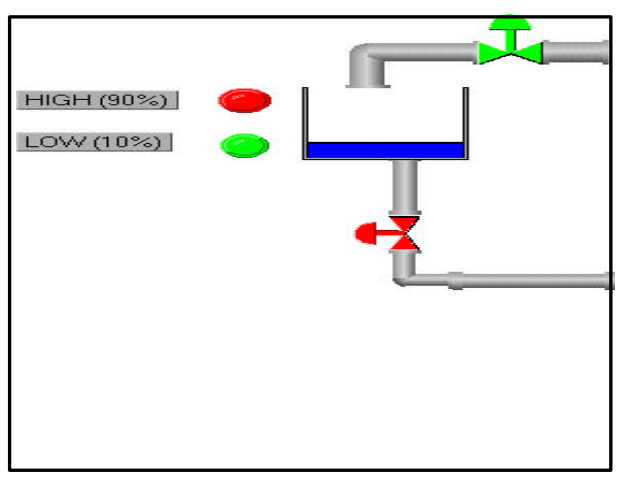

Figure 4 - A tank with two level sensors

With an extension of these rules we can control multiple micro-world cases like the one of five tanks (Figure 1)

In Figure 5 we can observe four examples of action sequences on a valve by the human user ( for example V12) and by the SCR (for example V12AUT). Also the evolution of the height in the tank regulated by the valves is given (for example h2(t)).

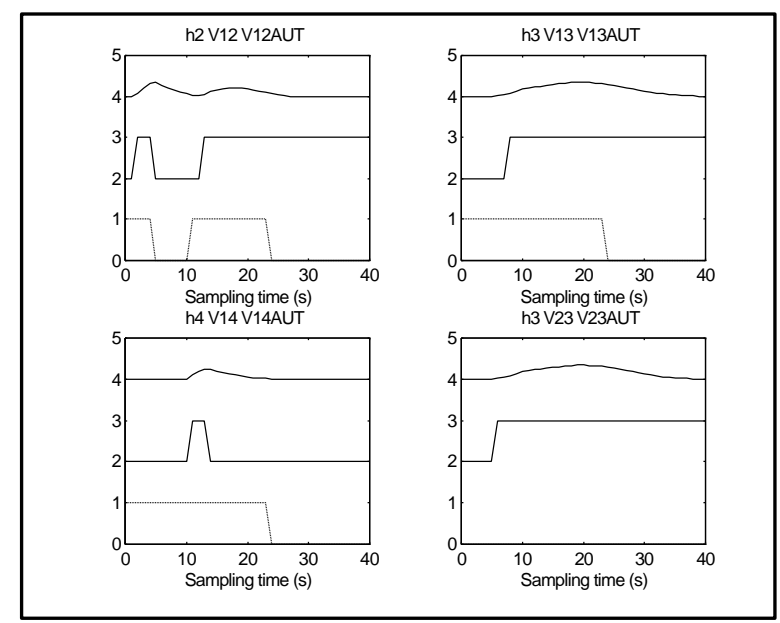

Figure 5 - Comparison between human and artificial actions 
If we observe the graphics of Figure 5, it is easy to see that a direct comparison between the AR actions and the human actions in order to extract information about behaviour, is a hard task and maybe an impossible task. The problems involved in this comparison are:

- the actions (open/close valves) can be done at any time

- the duration of the test can be different

- the decision sequences can be totally different and with the same successful supervision

Therefore, a more complete mode of comparison is necessary.

\subsection{Implementation of a qualitative RAR}

A qualitative RAR (QRAR) with two variations has been created. The variations correspond to a Nä̈ve Qualitative Agent (NQA) and a Qualitative Agent (QA) [8].

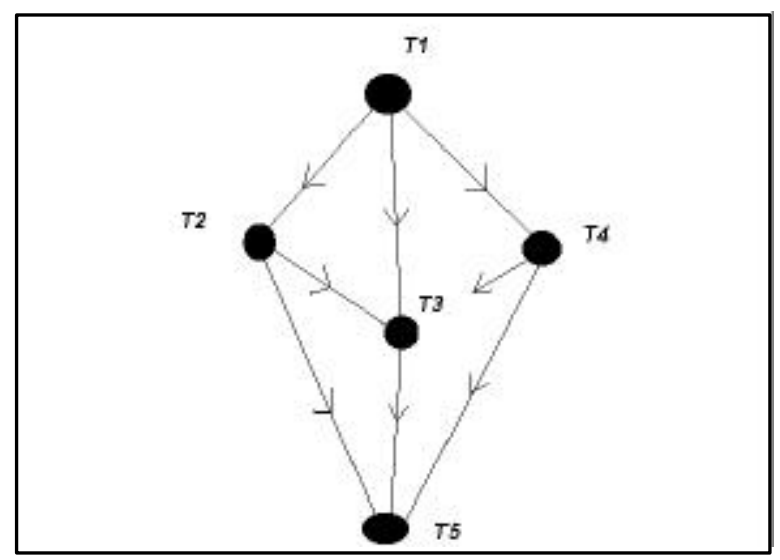

Figure 6 - The oriented graph (from micro-world of Figure 1)

From the point of view of the qualitative RAR, the micro-world can be formally represented by an oriented graph $\mathrm{G}=(\mathrm{V}, \mathrm{A})$. The set of vertices $\mathrm{V}=\{\mathrm{T} 1, \mathrm{~T} 2, \mathrm{~T} 3, \mathrm{~T} 4, \mathrm{~T} 5\}$ corresponds to the tanks and the set of $\operatorname{arcs} \mathrm{A}=\{(\mathrm{Ti}, \mathrm{Tj})\}$ corresponds to the set of pipes. (Figure 6).
The instruction is the following: "move water from top tank to bottom tank with minimal time and without water overflow".

The $N Q A$ and $Q A$ distinguish two cases in the decision-making procedure: case without alarms and case with alarms. A set of four goals has been defined [8]:

- (G1) do not enlarge any alarm

- (G2) reduce the number of alarms

- (G3) achieve and maintain an increasing tendency for the top tank

- (G4) maximise the number of open paths from the top tank to the bottom tank

In the first case, only (G3) and (G4) are retained whereas all four goals are used in the second case.

In the later case for instance, in mind we can give a grade for each admissible action as a function of goal success proportion. A linear weighted combination of the grade leads to the definition of a global grade. $(\mathrm{G})$

$$
\mathrm{G}=\mathrm{p} 1 * \mathrm{G} 1+\mathrm{p} 2 * \mathrm{G} 2+\mathrm{p} 3 * \mathrm{G} 3+\mathrm{p} 4 * \mathrm{G} 4
$$

The previous definition allows us to explore new means for behaviour comparison.

Connection between the LabVIEW microworlds generator and the qualitative reasoner

Data acquisition in LabVIEW is created by an internal function VI, where for each sampling time, it is possible to save each variable and also display the evolution of height during the test.

On the other hand, LabVIEW has two possibilities to save data into archive files: *.mat, and *.txt. The first one translates information into MATLAB format and it is useful for statistical analysis and mathematical data processing. The second one translates information into the program Mworld.exe. This last possibility allows the connection of LabVIEW micro-worlds and the qualitative RAR (Figure 7) 
These qualitative agents have been recently implemented in $\mathrm{C}++$ (Figure 7). We are working with:

- Input file (data.txt): a file with the topology of micro-world (number of tanks, number of pipes)

- Program Mworld.exe: the user chooses the reasoner and then the program Mworld.exe calculates the global grade for human action $G_{H}$ and for artificial action $G_{A}$

- Output file: it gives a file with the result of the comparison of human and reasoner actions at each instant.

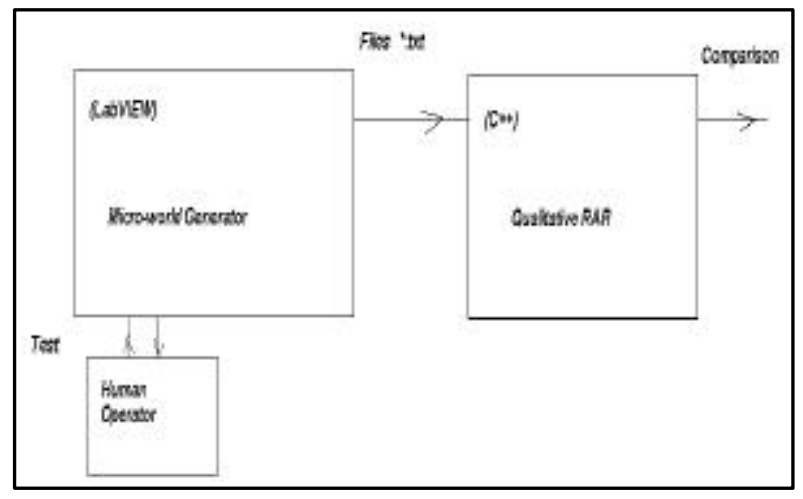

Figure 7- Connection between micro-world generator and RAR

\section{Testing and validation of the qualitative reasoners}

Two problems arise when we try to compare human test data and RAR test data, the temporal label problem and the local distance.

\subsection{Temporal label problem}

In previous work [8] it is stated that:

"The test session is organised so that, at each time instant, the human and the artificial operator make a decision on the next control action and these actions can be compared directly"
Because of the reaction time of the human operator, the action decided upon the state of the system at time $t(S(t))$ is recorded at time $(t+1)$ as $H(t+1)$. The same needs to be done for the QRAR actions if we want to be consistent in comparing the actions with similar temporal labels.

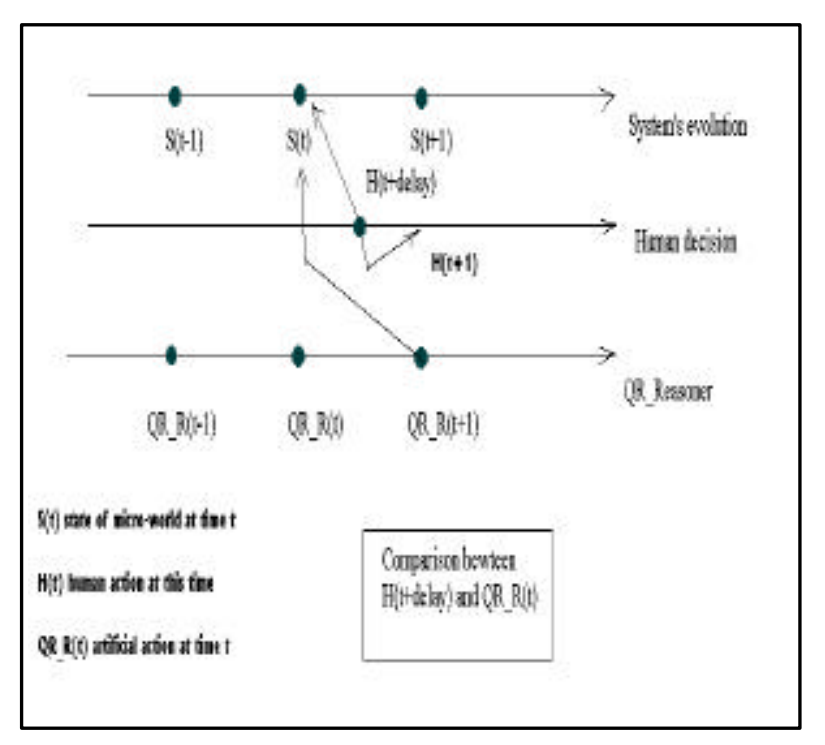

Figure 8 - Shift in the comparison of human and RAR actions

\subsection{Local distance}

The local distance that has been defined in [8] is currently tested. It is planned to use part of the set of data collected from the human sessions on the micro-world of Figure 1 to evaluate the soundless of the defined distance.

The distance is the following:

- Global grade $\mathrm{Ga}(\mathrm{t})$ and $\mathrm{Gh}(\mathrm{t})$ for the RAR and the human at time $t$

- Difference vector: $\mathrm{D}(\mathrm{t})=|\mathrm{Ga}(\mathrm{t})-\mathrm{Gh}(\mathrm{t})|$

- Distance:

$$
d=\sqrt{\frac{\sum_{j=1}^{m}\left(D t_{i j}\right)^{2}}{m}}
$$


The above distance gives a unique value for the comparison of a sequence of actions. Therefore, it is an accumulative value, and it is possible that two different users achieve the same distance with a different behaviour. For example, one user could have at the beginning of the sequence a totally different grade, then the RAR and exactly the same grade for the following actions. Another user could have, during all the test session, a slightly different grade. With the above definition of distance it is impossible to distinguish between them. And it is clear that they have different reasoning styles.

For example, in Figure 9 we have the plots of $G h(t)$ of four users with different behaviour:

- (Left top) $d=1$

- (Right top) $d=1.33$

- (Left bottom) $d=1.21$

- (Right bottom) $\mathrm{d}=2.0$

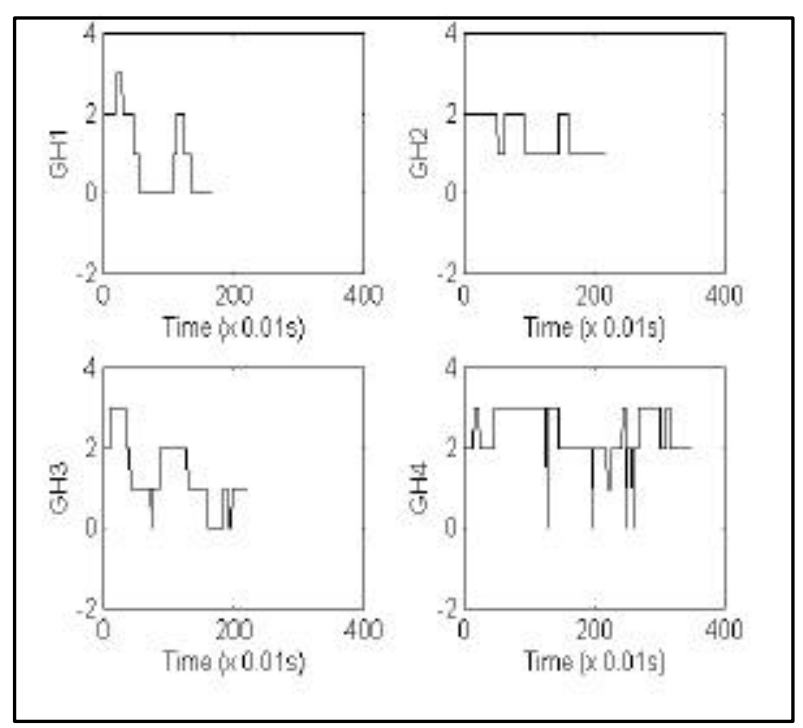

Figure 9 - Human grade versus time (artificial grade is zero)

\section{Proposition of future re- search lines and applica- tions}

The implementation of proposed agents (NQA and QA) is currently completed and tested. This step will be followed by a series of tests on human subjects and the evaluation of the implemented cognitive style in terms of their cognitive plausibility. This will eventually require the tuning of several of the RAR' parameters.

The classification of different human behaviours based on the proposed evolution of the grades it is possible to process by a neural network appproach (like Radial Basis Function Networks).

On the other hand, it is possible to design an artificial reasoner based on neural networks. In that case it is necessary to have an extensive data base with different cognitive styles and the help of an expert that will act as a supervisor . Trough this information we could modelize representative behaviours.

Some possible applications are:

a) Medical application: Cognitive assessment of patients with Parkinson's disease

This patient presents a "frontal syndrome" [1]. The set of cognitive impairments found in the frontal syndrome includes, for example, deficits in tasks requiring:

- Temporal ordering

- Planning

- And sequencing.

The issue is to acquire best knowledge of patient's behaviour in front of dynamic situations

a) Industrial application: Cognitive assessment of plant operators in the control room 
Operators most of the time are dealing with making decisions, anticipating events and giving diagnosis [10].

The issue can be to build a system which helps operators in making their decisions (behavioural cloning) [11] or to detect fatigue or stress situations.

\section{References}

[1] J. Pastor, A. Agniel, P. Celsis. Artificial Reasoners for the Cognitive Assessment of Patients with Parkinson's disease. ECAI98, $13^{\text {th }}$ European Conference on Artificial Intelligence, John Wiley and Sons, August 1998 Brighton

[2] A. M. Lázaro. LabVIEW. Programación gráfica para el control de instrumentación. Editorial Paraninfo, 1996.

[3] R.L. Mott. Mecánica de fluidos aplicada. Editorial Prentice-Hall Hispanoamericana, s.a. 1996.

[4] A.Creus Instrumentación industrial. Publicaciones Marcombo, s.a. 1979.

[5] The Math Works Inc. SIMULINK Dynamic System Simulation Software. User's Guide, Editorial Prentice-Hall International (UK) Ltd. 1997.

[6] B. Pasquet. Simulation par reseau causal qualitative d'un processus de surveillance optimal utilise comme reference dans l'evaluation $d u$ raisonnement d'un operateur humain. DEA Représentation de la Connaissance et Formalisation du Raisonnement. Université Paul Sabatier, Toulouse, septembre 1995

[7] P. Ponsa, A. Català. Human supervision in industrial process. $7^{\text {th }}$ IEEE International Conference on Emerging Technologies and Factory Automation. Barcelona, 18 to 21 october 1999.
[8] L. Travé-Massuyès, F. Prats, M. Sánchez, N. Agell, J. Pastor. A qualitative agent approach for assessing dynamic process human supervision. QR'99, The Thirteenth International Workshop on Qualitative Reasoning page 221. Loch Awe, Scotland, 6-9 June 1999

[9] L. Travé-Massuyès, P. Dague, F. Guerrin. Le raisonnement qualitatif pour les sciences de l'ingénieur. Editorial Hermes, Paris, 1997

[10] L. Leyval, S. Gentil, S. Feray-Beaumont. Model-based causal reasoning for process supervision. Automatica, Vol 30, No 8, pp1295-1306, Pergamon , 1994 Great Britain

[11] I.Bratko, T.Urbancic, C. Sammut. Behavioural clonning of control skill. From Machine Learning and Data Mining: Methods and Applications. Editat per R.S. Michalski, I. Bratko i M. Kubat. John Wiley and Sons, 1997

[12] H. Eyrolle, C. Mariné and S. Mailers. La simulation des environnements dynamiques: intérêts et limites. Paper from J. Cellier, V. De Keyser, and C. Valot. La gestion du temps dans les environnements dynamiques. Presses universitaries de France, Paris, 1996

[13] A. Cleermans, A. Destrebecqz, M. Boyer. Implicit learning: news from the front. Trends in Cognitive Sciences, Vol. 2, No 10, October 1998 